\title{
Culturalisme et développement à Vanuatu. Priorités, appropriations et savoirs légitimes
}

Culturalism and Development in Vanuatu: Priorities, Appropriations and Legitimate Knowledge

\section{Antoine HOCHET}

\section{OpenEdition}

\section{Journals}

Édition électronique

URL : http://journals.openedition.org/jso/7628

DOI : $10.4000 /$ jso. 7628

ISSN : $1760-7256$

Éditeur

Société des océanistes

Édition imprimée

Date de publication : 31 décembre 2016

Pagination : 205-222

ISSN : 0300-953x

Référence électronique

Antoine HOCHET, «Culturalisme et développement à Vanuatu. Priorités, appropriations et savoirs légitimes », Journal de la Société des Océanistes [En ligne], 142-143 | 2016, mis en ligne le 31 décembre 2018, consulté le 20 avril 2019. URL : http://journals.openedition.org/jso/7628 ; DOI : 10.4000/ jso.7628 


\section{Culturalisme et développement à Vanuatu. Priorités, appropriations et savoirs légitimes}

par

Antoine HOCHET ${ }^{*}$

\section{RÉSUMÉ}

Cet article esquisse une analyse des enjeux de l'aide publique au développement à Vanuatu, micro-Etat insulaire du Pacifique parmi les plus aidés au monde par habitant. L'analyse des effets de l'aide a nourri une littérature pléthorique souvent sujette à controverse dans les champs académique, politique et médiatique, notamment en accordant un poids important à linfluence des cultures locales. L'approche socioanthropologique employée ici conduit à dépasser l'explication culturaliste pour analyser l'impact de cette aide en se concentrant davantage sur la façon dont les priorités des donneurs influencent celles des récipiendaires de l'aide, en insistant sur les "effets secondaires" des projets de coopération. Cette perspective permet de modéliser la question de l'appropriation de l'aide dans une région où les régimes de savoirs des experts du développement s'avèrent difficilement accessibles pour les populations locales.

Mots-CLÉs : Aide au développement, culturalisme, anthropologie du développement, connaissance, changement climatique, paradoxe institutionnel de l'aide, Vanuatu

En décembre 2013, à l'Université de Technologie de Sydney, lors d'un déjeuner informel succédant à une matinée de conférences qui regroupaient de nombreux scientifiques du Pacifique et d'Europe, une discussion animée entre deux anthropologues, tous deux spécialistes de la Mélanésie, concentrait

\begin{abstract}
This article aims at drafting an analysis of the issues of official development aid in Vanuatu, Pacific Island Micro-State among the most assisted per capita in the world. The analysis of aid's effects has fed a large literature, often controversial within the academic, political and mediatic fields, in particular by granting an important weight to culture. The socio-anthropological approach being used here will firstly lead us to overcome the culturalist explanation to analyze aid effectiveness. The focus will be on the way donors priorities influence those of beneficiaries by highlighting their «side effects". This perspective will allow us to modelize the issue of aid appropriation in a region where knowledge regimes of development's experts turn out to be hardly accessible for local people.
\end{abstract}

KeYwords: Development aid, culturalism, anthropology of development, knowledge, climate change, aid institution paradox, Vanuatu

l'attention des invités $(17 / 12 / 2014)^{1}$. L'un d'entre eux, M. Harry ${ }^{2}$, est un anthropologue formé à Cambridge, ancien directeur d'une institution culturelle dans le Pacifique et désormais à la retraite. Son interlocuteur, M. Balley, professeur d'anthropologie dans une université australienne,

1. Je remercie Isabelle Leblic, Ludovic Levasseur, Denis Monnerie, Jean-Pierre Olivier de Sardan et Marc Tabani pour leurs remarques.

2. Les noms des deux anthropologues ainsi que les propos restitués par d'autres interlocuteurs dans notre article sont couverts d'anonymat. L'anonymisation fut une conditionnalité déterminante pour l'accord de nombreux entretiens. Toutefois, une minorité d'interviewés ont donné leur accord afin que leurs noms soient retranscrits, ils seront donc expressément nommés.

* Docteur en anthropologie, anhochet@gmail.com 
assume des fonctions de consultant pour le compte d'AUSAID (Australian Agency for International Development), du gouvernement australien, de la Banque mondiale et d'autres importants bailleurs de fonds internationaux. Cet anthropologue dispose d'une forte légitimité institutionnelle comme spécialiste du droit foncier mélanésien et du développement dans le Pacifique. Il est également consultant sur les impacts sociaux de l'industrie de l'extraction minière et expert de la gouvernance et des politiques traditionnelles mélanésiennes. Les deux hommes se connaissaient indirectement de réputation.

Cet échange mettait en relief des prises de positions diamétralement opposées concernant les enjeux qu'induit toute aide au développement dans les pays les moins avancés, à savoir : pour ou contre une intervention des pays développés dans les pays les moins avancés. Les arguments exposés étaient également caractéristiques des deux principales approches antagonistes défendues dans les milieux académiques sur la question.

La première d'entre elles critique la dimension intrusive des pays donataires dans les affaires intérieures des pays bénéficiaires et ses conséquences indésirables. Un des arguments de M. Harry était que les Mélanésiens, notamment les $\mathrm{Ni}$-Vanuatu, n'avaient pas, ou très peu, besoin de l'argent de l'aide étrangère pour assurer leur bien-être ${ }^{3}$. Par sa longue expérience dans l'archipel, il affirmait d'ailleurs qu'en zone rurale, la plupart des habitants ${ }^{4}$ ne bénéficie pas, de facto, d'aide au développement. Il avançait encore que les $\mathrm{Ni}$-Vanuatu vivent le plus souvent dans des conditions autarciques, en autosubsistance, sans intervention de l'État, ou plus généralement de toute autre institution moderne et enfin, que ces communautés rurales insulaires s'étaient auto-suffit depuis des millénaires. Selon lui, les donneurs, par leurs interventions et malgré les bonnes intentions affichées, produisent surtout des effets dérégulateurs, voire ils génèrent des conséquences néfastes sur les structures coutumières locales.

M. Harry défendait également l'idée qu’à long terme ces interventions extérieures, qui promeuvent un développement économique capitaliste moderne, incitaient les populations à quitter un mode de vie et un système horticole traditionnels qui garantissent aujourd'hui encore une situation où personne ne souffre de famine dans ces zones. L'emprise d'un travail salarié leur permettrait à peine de gagner un revenu suffisant pour subvenir aux besoins d'un foyer, ou ne serait-ce que pour prendre en charge un loyer.
L'autre alternative ne pourrait donc être que le renforcement du recours aux formes les plus précaires de salariat, puisque dans leur majorité, ces populations rurales ont peu de qualifications et manquent d'expériences professionnelles pour accéder à des postes à responsabilité.

Face à ces arguments, M. Balley défendait l'idée que, parfois, certains aspects des traditions des sociétés mélanésiennes contribuent à compromettre leur bien-être, notamment lorsqu'il s'agit de communautés isolées, peu monétarisées et qui disposent d'un accès limité aux infrastructures de santé ou de transports. Dans ce cadre, selon ce professionnel du développement, la culture mélanésienne constituerait un frein sinon un obstacle au bien-être et au développement des populations. Il illustra ses propos en s'appuyant sur des situations qu’il avait déjà observées :

«lorsqu'il n'y a pas d'hôpital près de ces villages, si quelqu'un était malade, si un enfant l'était, on le laissait mourir. C'est une situation qui n'est pas admissible et encore moins acceptable. » (Extrait du dialogue entre M. Harry et M. Balley)

Avec cet exemple, l'anthropologue consultant argumentait donc que le dénuement des populations justifiaitl'intervention des donneurs. En réponse à ces propos, $M$. Harry évoqua alors l'usage de la médecine traditionnelle - la kastom meresin - et de ses capacités à traiter nombre d'afflictions. À titre d'exemple, il mentionna le village de Yakel à Tanna où la population a fait le choix d'affirmer une vision traditionaliste de son rapport au passé et où ce type de médecine est pratiquée. Suite à cet argument, M. Balley soupira et leva les yeux au ciel pour ostensiblement exprimer son exaspération. Il mit unilatéralement fin à la discussion en se levant et quitta la table. Ces deux professionnels ne se sont ensuite plus adressé la parole.

L'intérêt de restituer cette controverse tient en ce que chacun des deux interlocuteurs eut recours à un large éventail de registres justificatifs pour défendre leurs positions - pour ou contre l'aide au développement. L'un et l'autre invoquait successivement des arguments que l'on peut qualifier de culturaliste, de misérabiliste, de progressiste ou encore, de critique vis-à-vis de l'asymétrie des rapports de forces entre des donneurs, qui disposent de moyens d'action considérables, et les récipiendaires de l'aide.

Ce dernier argument nous intéresse particulièrement car il met en exergue le monopole des ressources matérielles, et surtout symboliques des

3. Selon la Banque mondiale, Vanuatu est classé comme le $12^{\mathrm{e}}$ pays le plus aidé au monde par habitant en aide publique au développement. Bien que l'archipel doive sortir de la liste des pays les moins avancés (PMA) en 2016, le budget d'aide publique au développement qu'il reçoit annuellement ne cesse d'augmenter depuis la mise en ouvre de son plan de réforme structurelle de 1997 (voir http://donnees.banquemondiale.org/ indicateur/dt.oda.odat.pc.-zs/countries).

4. Selon le Bureau national des statistiques du Vanuatu, en 2009, plus de $75 \%$ de la population vanuataise vit en zone rurale (Vanuatu National Statistical Office, 2011) 
donneurs tels les savoirs " pratiques " et légitimes 5 . Ces ressources, pour la plupart monopolisées par un type particulier d'agents du développement, souvent qualifiés d'«experts», jouent selon nous un rôle déterminant dans l'orientation des politiques et dans l'attribution des fonds publics. M. Balley étant d'ailleurs régulièrement contracté en qualité d'expert, la discussion retranscrite entre les deux anthropologues portait donc implicitement sur sa légitimité à appuyer les politiques extérieures de son gouvernement en se basant sur ses compétences de spécialistes des "cultures" et des institutions de l'aide au développement ${ }^{6}$.

Cet article discutera tout d'abord la pertinence de l'approche culturaliste pour expliquer l'absence de "développement". Ce débat majeur chez les anthropologues africanistes sera ensuite transposé au sein des discussions sur la question chez les océanistes. Les conclusions de ce débat seront alors confrontées à des observations conduites à Vanuatu (Mélanésie) où nous avons effectué plusieurs séjours ${ }^{7}$ et avons procédé à des séries d'entretiens avec des professionnels et experts impliqués dans le secteur de la coopération au développement ${ }^{8}$. Ces observations de terrain mettront à jour une asymétrie de pouvoir (financier, logistique, symbolique) entre donneurs et récipiendaires. Cette perspective nous conduira enfin à proposer une modélisation de trois types de réactions des structures locales vis-à-vis de l'aide, ceci en interrogeant les phénomènes d'appropriation, d'efficacité, mais également, de résistances vis-à-vis de certains projets soutenus par des donneurs aux ressources surdimensionnées à l'échelle du pays et omniprésents dans les administrations publiques.

\section{Le culturalisme populiste et misérabiliste}

La culture comme principal facteur explicatif de la pauvreté des populations dans les pays les moins avancés, et par extension, des dysfonctionnements des appareils d'État dus à une "mauvaise gouvernance" de leurs administrations ou encore de la propension à la corruption des élites, suscite de nombreux débats scientifiques et politiques. Une des dernières contributions en date est celle d'Olivier de Sardan, intitulée Le Culturalisme traditionaliste africaniste [CTA]. Analyse d'une idéologie scientifique (Olivier de Sardan, 2010). Dans cet article, l'auteur développe une critique d'un type particulier de culturalisme: le culturalisme "savant" qu'il propose de dépasser grâce à une méthodologie dite socio-anthropologique centrée sur l'agencéité des acteurs et l'hétérogénéité des logiques sociales s'imbriquant en "situation de développement" - méthodologie vers laquelle nous tendons. Selon Olivier de Sardan :

" les postulats de base de ce culturalisme savant sont relativement simples. Le faible respect en Afrique des règles $\mathrm{du}$ jeu formelles serait dû au poids des pratiques informelles, d'origines sociale et culturelle, qui s'inviteraient en permanence dans les dispositifs étatiques. La 'pression communautaire', les coutumes locales, les valeurs traditionnelles, les représentations magico-religieuses, les habitudes clientélistes et patrimonialistes, les solidarités primordiales, les identités ethniques sont ainsi régulièrement invoquées. Seule une analyse de la culture africaine, et de son ancrage dans le passé, pourrait permettre de comprendre les pratiques politiques en vigueur»(Ibid. : 422).

Olivier de Sardan développe une argumentation critique à l'égard de cette vision figée, abstraite, réductrice et généralisante d'une culture africaine uniforme et unanimement partagée à travers le continent. Il prend comme point de départ le concept de "matrice morale " spécifiquement africaine développée par Schatzberg (2001) et présentée comme :

"sous-jacente aux comportements politiques, ceux des élites comme ceux des agents de l'État ou même

5. Nous faisons ici référence aux savoirs pratiques tels qu'évoqués par Malinowski pour appuyer les administrateurs des anciennes colonies. Malinowski appelait au développement d'une " anthropologie pratique " (practical anthropology) du changement des modes de vie des peuples colonisés pour soutenir les « hommes de terrain " (practical man) (Malinowski, 1929 : 36).

6. Cette position d'anthropologue consultant pose en effet des questions éthiques pour le chercheur, sans doute davantage lorsqu'il travaille pour un gouvernement qui intervient en dehors de ses frontières. Le recrutement d'anthropologues pour former ou assister des troupes américaines en Afghanistan ou en Irak met en lumière les usages des plus controversés de la coopération institutionnelle entre sciences sociales et gouvernements. Voir l'analyse proposée par Julien Bonhomme à propos d'anthropologues « embarqués » (Bonhomme, 2007).

7. Notre terrain à Vanuatu s'est déroulé en deux périodes. La première de septembre 2010 à février 2011 (6 mois) principalement sur l'île d'Espiritu Santo ; la seconde de janvier 2012 à octobre 2013 où nous travaillions pour le Centre Culturel du Vanuatu, à Port-Vila. Nous avons ainsi pu observer au quotidien les enjeux de la coopération scientifique et culturelle au sein de cette agence gouvernementale de la culture et de la recherche scientifique qui met en œuvre de nombreux projets financés par une grande diversité de bailleurs de fonds (Banque Mondiale, Union européenne, AUSAID, Agence Française de Développement, UNESCO, etc.).

8. Quarante-deux entretiens formels ont été enregistrés et 21 dialogues informels ont été notés. Huit réunions impliquant des agents impliqués dans des projets de développement ou de coopération scientifique ou culturelle ont été enregistrées À cela s'ajoutent 1641 articles de presse scannés (Dailypost, seul journal quotidien en support papier de l'archipel), douze débats radiodiffusés traitant de la culture vanuataise (Tokbak Show), de nombreux courriels et documents internes des institutions et des professionnels du développement sur la mise en œuvre de projets furent également collectés. 
des simples citoyens [et] définirait un cadre cognitif commun pour une société tout entière. " (Ibid. : 215) ${ }^{9}$

Cette matrice morale reposerait sur un socle de valeurs, de symboles et de significations partagés, notamment en ce qui concerne les manifestations de la légitimation du pouvoir par les responsables politiques africains. Ces derniers useraient abusivement de l'emploi de métaphores paternalistes, familiales et relatives à la nourriture qui selon Schatzberg tireraient leurs origines d'un univers symbolique hérité d'un passé précolonial faisant particulièrement sens en Afrique.

Olivier de Sardan explique cette propension à réifier le concept de culture de façon vague ${ }^{10}$ par l'influence de la pensée de Talcott Parsons. Cette explication fut également avancée par Adam Kuper (1999) qui analyse historiquement et comparativement l'évolution du concept de culture dans le champ des sciences sociales. Selon Kuper, Parsons participa à ce que la culture devienne " un terme 'parapluie' pour tout ce qui concerne les idées et les valeurs » (1999: 53). Mais, pour Olivier de Sardan, la récurrence du recours à la culture comme "system of meanings ", s'avère

" en fait, dans les usages qu'en font tous ceux qu'y s'y réfèrent ${ }^{11}$ un concept flou, polymorphe, aussi général qu'imprécis, de type "auberge espagnole"." (Olivier de Sardan, 2010:437)

Il propose alors « un usage raisonné, circonscrit et empiriquement attesté » de la notion de culture en réintégrant la définition originelle et subsystémique de Tylor selon laquelle la culture serait :

« ce tout complexe comprenant à la fois les connaissances, les croyances, les arts, la morale, les lois, les coutumes et les autres facultés et habitudes acquises par l'homme dans l'état social. » (Tylor, $1871: 1$ )

Olivier de Sardan insiste enfin sur l'aspect dynamique, hétérogène et multidimensionnel des manifestations de traits culturels spécifiques aux sociétés africaines. Il réfute donc l'idée de "commonality" présentée par Goody comme un "système de significations et de valeurs partagées" (Goody, 1992 : 18), ou en d'autres termes, d'un socle commun, cristallisant une "culture africaine commune " indifférenciée de l'ensemble de la population du continent.

Pour remédier à ces imprécisions, l'auteur préconise d'une part, de réintroduire l'analyse des "pratiques» directement observables, et d'autre part, de mettre à jour

"le concept de représentations sociales [qui] a l'avantage de rester proche des discours proférés et se fonde sur ceux-ci : c'est ce en quoi il est empiriquement attestable et relève de l'émique, à la différence de concepts tels que "valeurs", "visions du monde", "matrice morale", "systèmes de sens", etc. " (Goody, 1992: 442)

Pour démontrer la fécondité de cette approche méthodologique, Olivier de Sardan s'appuie sur des enquêtes approfondies menées notamment en Afrique de l'Ouest francophone depuis les années 1990

"sur des cultures professionnelles, religieuses, locales, générationnelles, de genres, politiques ou institutionnelles nettement circonscrites [qui] contredisent les clichés et font voler en éclats les présupposés culturalistes. " (Olivier de Sardan, 2010 : 437) ${ }^{12}$

Cette critique du culturalisme se doit enfin d'être intégrée dans deux perspectives qui participent à la même dynamique d'essentialisation de la culture. La première, qualifiée de "populiste ", valorise les cultures et leurs résiliences. Lors de l'échange entre les deux anthropologues retranscrit plus haut, M. Harry adoptait cette posture. Il mettait effectivement en avant la résilience séculaire des modes de vie des communautés mélanésiennes et argumentait pour leur préservation ainsi que pour leur promotion. La seconde, qu'il est possible de qualifier de "misérabiliste" (Grignon et al. : 1989), est davantage employée par les professionnels du développement. Ils ont ainsi recours à des valeurs morales pour inciter et légitimer l'intervention extérieure afin de rétablir la «dignité » des populations. M. Balley

9. D’après Schatzberg, « cette matrice est réellement une série de prédispositions culturelles et de compréhension implicite qui façonne une structure sous-jacente aux lexiques politiques, aux concepts, aux images, aux institutions et aux comportements que nous considérons comme pensables et donc légitimes » (Schatzberg, 2001: 215).

10. Expression empruntée à Olivier de Sardan qu'il emploie en référence à la notion de "quantificateur vague " de Passeron (1990).

11. Cet ensemble de valeurs, symboles et significations constituerait alors à " une structure de significations " homogène à travers laquelle les gens donnent forme à leur expérience (Schatzberg, 2001 : 4). Schatzberg fait ici directement référence à une définition de la culture telle que proposée par Geertz, selon laquelle, la culture est un "système ordonné de significations et de symboles...en fonction desquels chaque individu définit son monde, exprime ses sentiments et forme son jugement " (Geertz, $1973: 68)$.

12. Pour étayer son argumentation, Olivier de Sardan s'appuie sur les travaux menés depuis les années 1990 par l'Association euro-africaine pour une anthropologie du changement social et du développement (APAD) qui remet en cause les clichés et stéréotypes d'agents du développement sur l'explication monocausale culturaliste dans différentes micro-cultures sectorielles telles que dans la santé (Jaffré et Olivier de Sardan, 2003), les corps paramilitaires des eaux et forêts (Blundo, 2011a) ou des douanes (Cantens, 2009), etc. Pour une même critique sur un terrain océaniste (en Nouvelle-Calédonie), voir Leblic (1993). 
utilisait ce type d'arguments culturalistes qui tendent davantage à se concentrer sur les critères anomiques des sociétés mélanésiennes ou leur inadéquation avec la " modernité ». Ainsi, ce qui apparaissait au départ comme deux positions à l'antagonisme insurmontable relèvent finalement d'une même logique culturaliste.

\section{Un culturalisme traditionaliste océaniste ?}

La critique du culturalisme en Afrique pourrait s'appliquer aux thèses du politiste Benjamin Reilly qui dresse quatre constats sur l' " africanisation du Pacifique Sud » :

a) les tensions croissantes entre les régimes civils et les forces militaires

b) un mélange entre les identités ethniques et le processus de compétition pour le contrôle des ressources comme facteur de conflits

c) la faiblesse des institutions de base telles que les cabinets ministériels, les parlements ou encore les partis politiques et enfin,

d) l'importance croissante du rôle de l'État comme moyen d'obtenir des richesses et d'accéder et exploiter ses ressources (Reilly, 2000 : 63).

Dans un autre article (Reilly et al., 2002), Reilly développe avec plus de précision l'idée que le tribalisme, le factionnalisme ou encore le clientélisme électoral ont en commun d'être basés sur une "identité ethnique ", concept emprunté à l'identité "primordiale" de Geertz (1973) même si l'auteur reconnait les limites heuristiques de cette notion d'identité ethnique basée sur l'appartenance linguistique, géographique, familiale ou clanique (Reilly, 2002:915).

Afin de démontrer le primat du facteur ethnique dans les stratégies d'accès aux fonctions de l'Etat, Reilly a recours à des données sur l'origine des membres du parlement de Papouasie Nouvelle-Guinée. Il remarque tout d'abord une certaine vitalité du processus démocratique dans le turn-over parlementaire. Il explique cette situation par la grande diversité culturelle papoue néo-guinéenne qui empêche toute ethnie démographiquement majoritaire de prendre les rênes du pouvoir au détriment des minorités. Reilly souligne toutefois que ce dynamisme n'est pas exempt de logiques patrimonialistes où selon lui, le pouvoir des big men définit la loi (Reilly, 2002: 925). Ces prétendants aux pouvoirs auraient recours à la mobilisation de valeurs et de symboles basés sur l'identité ethnique afin de fédérer leur électorat et ainsi accéder aux ressources qu'offrent les postes gouvernementaux. Cette configuration mènerait selon lui à une concurrence exacerbée entre "clans » et un factionnalisme nocif pour la redistribution des richesses et, in fine, pour le développement général du pays.

Cette idée que l'appropriation par les élites politiques des ressources de l'État suive des logiques culturelles claniques et factionnalistes n'est pas nouvelle. Elle entre effectivement en résonnance avec d'autres analyses essentialistes et généralisantes de la " politique du ventre " (Bayart, 1989) également appliquées à l'Afrique où l'appareil étatique est accusé d'être " prébendier " (Joseph, 1987), "prédateur" (Darbon, 1990 ; Fatton, 1992), ou encore "kleptocratique" (Coolidge et Rose-Ackerman, 2000) ${ }^{13}$.

Outre le caractère provocateur, réducteur et difficilement défendable de la formule $d^{\prime}$ " africanisation du Pacifique ${ }^{14}$, cette perspective signale toutefois avec une relative justesse la centralité de l'État que nous avons également pu observer lors de notre terrain :

" à travers toute la région du Pacifique Sud, notamment en Mélanésie, l'accès à l'État est un (peut-être le) facteur crucial du succès économique, puisque l'État, et non le marché, est en lui-même l'instrument primaire pour l'accumulation des ressources telles que l'aide au développement étrangère et les revenus domestiques. " (Reilly, 2000 : 266)

Ces deux mannes financières, "aide au développement» et "revenus domestiques ", évoquées brièvement par Reilly, ont selon nous une influence prépondérante sur les agents et les institutions publiques des pays qui en bénéficient. Le choix de Reilly de se concentrer sur les logiques factionnalistes et prédatrices en fonction d'identités ethniques afin d'accéder à l'État occulte en effet les effets structurants de ces deux mannes " au quotidien ». L'aide apparaît pourtant comme un facteur tout aussi (sinon plus) déterminant que l'explication ethnique pour rendre compte du fonctionnement des États mélanésiens.

Cette inversion de perspective est d'autant plus pertinente que le Pacifique est une des régions les plus aidées au monde par habitant ${ }^{15}$ et bénéficie de l'intervention de donneurs d'une grande diversité - bien que l'Australie, parfois qualifiée de "gendarme du Pacifique " (Argounès, 2012) s'affiche comme le premier donneur régional.

Dans quelle mesure cette aide structure la gouvernance des pays du Pacifique et produit des effets indésirables? Pour répondre à cette question, définissons avant ce qu'est l'aide au développement.

13. Liste d'adjectifs qualificatifs déjà formulée par Blundo (Bierschenk et al., 2007 : 56).

14. Les principales critiques des thèses de Reilly sur l'africanisation du Sud Pacifique ont davantage été formulées par des anglophones (voir Chappell, 2005 ; Firth 2001 ; Fraenkel 2004). Voir aussi Leblic (1993).

15. Voir http://data.worldbank.org/indicator/dt.oda.odat.pc.zs (dernier accès 14/05/2015). 


\section{Développement : idéologie, industrie et "violence épistémique »}

Le terme "développement " peut caractériser soit un état, soit un processus. Il est le plus souvent mis en relation avec les concepts de "bien-être ", de "progrès ", $d$ ' "équilibres sociaux", de "croissance ", d' "épanouissement personnel ", des "enjeux écologiques", ou encore de la «bonne gouvernance " (Rist, 1996 : 19) ${ }^{16}$. D'après Serge Latouche, le concept de développement peut trouver ses sources

«dans les deux dimensions de la constitution de l'Occident lui-même : la dimension hellénique et la dimension judaïque. » (1989: 52)

La première le drape d'une certaine conception prométhéenne de l'homme, comme maitre de la nature grâce au calcul et à la raison. La seconde lui accorde la dimension d'une téléologie christique et sotériologique (Latouche, 1989: 52). La principale propriété du développement serait donc d'être un processus évolutif, inscrit dans le temps, tirant ses origines d'une histoire occidentale singulière et dont la finalité majeure serait d'être vouée à l'atteinte d'un but ultime : le bien commun de l'humanité.

Parmi les nombreuses définitions du développement, celle de Thomas Bierschenk retranscrit le caractère polysémique du terme en intégrant un angle d'analyse qui permet l'observation de ses manifestations. Il considère en effet le développement :

" comme un processus historique de longue durée, comme transformation structurelle d'une société incluant un questionnement sur les sources du changement social [mais également] comme "projet" politique et économique d'une époque donnée, à savoir la période qui fait suite à la Seconde Guerre mondiale, marquée par l'universalisation de l'idée de la possibilité de changements sociaux induits. " (Bierschenk, 2009:31)
L'anthropologue considère enfin toute action d'aide au développement comme :

" [une] situation sociale structurée par les interactions directes et indirectes entre acteurs et groupes sociaux dans un contexte de développement planifié, le projet de développement en constituant l'exemple paradigmatique " (Ibid.),

il serait donc observable d'après une méthodologie empirique.

Projet idéologique, politique et économique se matérialisant en situations sociales structurées par des interactions entre différents acteurs et groupes sociaux, le développement induit toutefois l'existence de son antonyme : le "sousdéveloppement ${ }^{17}$. Cette distinction participe alors à renforcer l'idée d'un Grand Partage (Great Divide) (Latour, 1983 ; Lenclud, 1992) entre les "développés ", les donneurs, et les populations qui ne le seraient pas, les récipiendaires de l'aide. Cette conception bipolaire, le plus souvent basée sur des critères économétriques et quantitatifs (PIB, IDH, points de croissance, etc.), faisant le lit de la célèbre dichotomie entre l'" Ouest et le Reste " (Sahlins, 1976), a pour effet de cristalliser l'idée d'un écart entre sociétés modernes, pourvoyeuses d'aides, riches et en majeure partie occidentales, et les autres, traditionnelles, aux faibles performances économiques qui, en termes maussiens, seraient également en position d' " infériorité " par leur impossibilité de rendre le don (Mauss, 1925: 90) ${ }^{18}$. Ces dernières n'auraient alors d'autre choix que de s'adapter aux modèles conçus par les "professionnels du développement $»^{19}$, principalement constitués d'experts, généralement occidentaux sinon, membres d'une élite locale très restreinte, formée dans des centres universitaires internationaux.

Cette appréhension duale de l'aide donneurs/ récipiendaires convient toutefois d'être nuancée car le monde du développement recouvre une hétérogénéité d'agents, de structures et de pratiques. Il est en effet composé :

16. Selon Rist, le «développement " peut également être défini dans des conceptions plus paradigmatiques en lien avec l'« évolutionnisme social » sinon l'individualisme (il s'agit de développer la personnalité des êtres humains), ou encore l'économie (croissance, accès au revenu, avec ses signifiés normatifs (ce qui doit arriver), instrumentaux (à quoi cela sert) ou suggérant des manques ou des défauts actuels (Rist, 1996 : 21). Voir aussi sur ces questions le chapitre d'Isabelle Leblic « Le développement en question" (1993: 10-21).

17. Rappelons que $75 \%$ des pays du monde bénéficient d'aide publique au développement. Voir la liste des pays récipiendaires de l'aide des 34 membres du Comité d'Aide au Développement de l'ocDE en 2013 : http://www.oecd.org/dacl stats/documentupload/dac\%20list\%20of\%20oda\%20Recipients\%202014\% 20final.pdf, dernier accès le 12/03/2015.

18. Selon Mauss, «le don non rendu rend encore inférieur celui qui l'a accepté, surtout quand il est reçu sans esprit de retour. [...] La charité est encore blessante pour celui qui l'accepte, et tout l'effort de notre morale tend à supprimer le patronage inconscient et injurieux du riche "aumônier" ». (Mauss, 1925: 90).

19. La modélisation des Étapes de la croissance économique des États nouvellement indépendants conçue par Rostow (1963) figure comme un exemple emblématique d'une conception évolutionnisme unilinéaire du développement qui a d'ailleurs fortement influencé la politique extérieure américaine depuis la fin de la Seconde Guerre mondiale (Park, 2006). Toutefois, l'attribution de l'aide a recouvert plusieurs formes depuis son institutionnalisation. L'économiste du développement Hugon distingue trois phases qui caractérisent les modalités de délivrance de l'assistance au développement depuis la fin de la Seconde Guerre mondiale. La première est celle de " radicalisation et d'affrontement " (1968-1980), de « la libéralisation et de l'ajustement " (1980-1995) et la période de « la refondation et du post-ajustement " (1995 à aujourd'hui) (Hugon, 2007). 
"[d'un] univers largement cosmopolite d'experts, de bureaucrates, de responsables d'ONG, de chercheurs, de techniciens, de chefs de projets, d'agents de terrain, qui vivent en quelque sorte du développement des autres, et mobilisent à cet effet des ressources matérielles et symboliques considérables. "(Olivier de Sardan, 1995: 7)

Ainsi, depuis la fin de la Seconde Guerre mondiale, le montant de l'aide publique au développement (APD) est constamment en augmentation et représente actuellement un budget annuel d'environ 140 milliards de dollars par $\mathrm{an}^{20}$. Le secteur de la solidarité internationale constitue alors pour certains une "industrie" (Lewis et Mosse, 2006: 6 ) voire un "business " (Birdsall, 2004 ; Moss etal.,2006) au sein desquels évoluent une myriade d'agents et d'institutions aux pratiques et aux compétences sectorialisées, de plus en plus techniques. Elles forment finalement des "communautés épistémiques" (Viltard, 2006) qui manipulent un langage, des logiques et des savoirs spécifiques à la gestion de projets et à la mise en ouvre d'action publique dans les pays en voie de développement.

Le monde de l'aide au développement s'avère donc historiquement structuré, institutionnalisé, professionnalisé et normalisé depuis plus d'un demi-siècle. Ce phénomène a conduit à ce que les acteurs majeurs de l'aide concentrent désormais leurs efforts autour d'objectifs mondialisés et de financements basés sur les résultats (tels que les objectifs du millénaire pour le développement) et a priori non plus sur les budgets engagés. Cette institutionnalisation de l'aide contribue également à ce qu'elle soit désormais devenue un secteur prépondérant dans le paysage de la coopération et des relations internationales. Mais l'analyse empirique de l'impact de cette aide reste peu étudiée. Elle constitue l'une des "zones d'ombre" des productions savantes sur la question, plus particulièrement lorsqu'il s'agit $d^{\prime}$ '" ethnographie des relations entre système de l'aide et structures étatiques des pays aidés " (Blundo, 2011).

Selon Blundo, l'ensemble des analyses macropolitiques existantes concernant les effets indésirables de l'aide sur les institutions publiques des pays récipiendaires peuvent se répartir en trois principales thématiques. La première est celle considérant que les institutions internationales (types Banque mondiale, FMI) destitueraient les États de leur souveraineté et participeraient alors à la pérennisation d'une néo-dépendance postcoloniale (Nugent, 2010 ; Bayart, 1999; Anders, 2005). La deuxième thématique se concentre sur «la capture de l'aide par des régimes politiques de types néo-patrimonial " (Blundo, ibid.). La manne financière de l'aide au développement est considérée comme une opportunité de ressources à capter pour les élites, captation qui amplifierait les phénomènes de patronage, de népotisme et de corruption, et qui, pour le Pacifique, fait écho à l'analyse développée plus haut par Benjamin Reilly. Enfin, la troisième thématique à partir de laquelle nous déploierons notre analyse sur les effets structurants de l'aide au développement à Vanuatu, repose sur l'idée que «les projets soutenus par des donneurs seraient un facteur de fragmentation des administrations postcoloniales " dans le sens où "la prolifération de donneurs et de projets constituent un fardeau substantiel pour le faible nombre de fonctionnaires locaux qualifiés qui passent le plus clair de leur temps à s'adapter aux priorités des donneurs et à gérer des activités d'aide au développement plutôt que de promouvoir le développement du pays quand ils ne quittent pas le service public pour une meilleure rémunération auprès de donneurs ou d'ONG " (Moss, Pettersson \& van de Walle $2006: 6$, trad. A. Hochet).

$\mathrm{Ce}$ "détournement de l'attention" des agents locaux du développement d'une promotion endogène des activités de leurs administrations, au profit des initiatives soutenues par les donneurs, qualifié d' "aid institution paradox", s'applique aisément au Vanuatu. L'archipel, classé au rang du $12^{\mathrm{e}}$ pays des plus aidés au monde par habitant, voit effectivement proliférer sur son territoire de nombreux projets et une grande diversité de donneurs qui mobilisent un nombre important de fonctionnaires.

Mais pour prendre part à ces nombreux projets de développement, ces agents locaux doivent disposer de connaissances et de compétences spécifiques, en accord avec les procédés de mise en œuvre de projet des donneurs ${ }^{21}$. En d'autres termes, il est nécessaire que ceux-là disposent de compétences propres à la culture professionnelle de plus en plus spécialisée du monde du développement.

20. Il convient toutefois de relativiser ce montant global de l'aide publique au développement en ce qu'elle peut davantage revêtir la forme d'aide "fantôme " telle que l'effacement des dettes des pays "aidés " ou encore d'aide "boomerang " dépensée dans les frais surfaits de prestations de consultance (Severino et Ray, 2011).

21. Le "cadre logique " apparaît comme un instrument clé et des plus représentatifs de ces logiques développementalistes. Conçu pour clarifier l'agenda et les objectifs des projets de développement, mais parfois complexes à échafauder. Le cadre logique est pourtant utilisé dans la quasi-majorité des projets d'aide au développement implantés dans les pays du Sud. Olivier de Sardan et Giovalucchi le décrivent en ces termes. «Le « cadre logique » est une technique de rationalisation de l'action publique centrée sur la planification par objectifs, devenue omniprésente dans les institutions de développement. Il privilégie une perspective purement gestionnaire centrée sur les facteurs quantifiables, la légitimation de l'intervention et une vision linéaire et mécanique de la causalité. Cette perspective ignore les facteurs politiques, les conflits, le rôle des acteurs ou les ajustements stratégiques. Elle repose sur une épistémologie positiviste largement dépassée, loin des acquis de 
Mais au vu des faibles performances du système éducatif local, encore peu de Ni-Vanuatu exercent ces fonctions d'expertises qui sont dès lors très régulièrement prises en charge par des personnels expatriés, le plus souvent, occidentaux. La figure centrale de l'expert se présente alors comme un rouage déterminant dans la mécanique de la logique de projet.

\section{Appropriation et rapports asymétriques aux savoirs légitimes}

Arturo Escobar est l'un des plus fervents critiques des politiques publiques impulsées par les grandes institutions internationales de la coopération et leurs agents. Ces organisations, notamment la Banque Mondiale et le PNUd, exerceraient une domination épistémologique à travers leurs "régimes de connaissances " spécifiques. Selon lui, «le développement a exclusivement reposé sur un seul système de connaissance, à savoir, l'Occidental » qui aurait "dicté la marginalisation et la disqualification d'autres systèmes qui ne seraient pas occidentaux " (Escobar 1995: 13). Ce positionnement critique vis-à-vis de la verticalité des politiques publiques du développement, basée sur la domination d'un régime de savoirs occidentalocentré, fut également mis en lumière par Mark Hobart (1993). Selon lui, la partition binaire des savoirs présentés soit comme scientifiques, soit comme traditionnels, serait nocive en ce qu'elle pérenniserait une logique de domination des savoirs des « développeurs".

"Les relations entre les "développeurs" et les "développés" seraient construites sur la base des catégories et des savoirs des "développeurs". La dimension épistémologique de tels processus et leurs liens étroits avec les phénomènes de pouvoirs, sont souvent marqués par les discours du développement exprimés principalement dans les langages de l'économie, de la technique ou de la gestion [...]. Les savoirs populaires sont ignorés ou considérés comme des obstacles au progrès rationnel." (Hobart, 1993 : 2).

Notre analyse ne s'attardera pas sur les « savoirs traditionnels " et leur prise en compte dans les projets de développement, mais se concentrera sur les "savoirs pratiques" des professionnels du développement qu'ils soient de nationalités étrangère ou vanuataise, et que nous qualifions de "ressources symboliques". Nous faisons ici référence au " capital symbolique " tel que défini par Bourdieu ${ }^{22}$. Cette inégalité de distribution des savoirs légitimes, majoritairement détenus par des étrangers ou les élites locales, n'est d'ailleurs pas sans rappeler le concept bourdieusien de "violence symbolique" ou encore, ses déclinaisons "subalternes" de "violence épistémique " (Spivak, 1999) voire d' "injustice épistémique" (Bhargava, 2013) à l'égard de certains groupes sociaux par " ceux qui savent". Appliqués au contexte vanuatais, les structures et les agents locaux du développement se verraient alors disqualifiés du processus de définition et de mise en œuvre des politiques publiques puisqu'ils ne manipuleraient pas ces savoirs légitimes propres aux modes de fonctionnement des organisations de l'aide internationale.

Selon nous, ce phénomène de mise à l'écart de "ceux qui ne savent pas" dans le processus de définition et la mise en œuvre des actions publiques contribue à creuser l" "implementation gap ${ }^{23}$ entre la conception d'un projet et ses résultats finaux. À cet égard, la question du manque d'appropriation par les structures locales des projets soutenus par les donneurs fut exposée de façon récurrente par nos interlocuteurs lors de notre étude sur le terrain. Cet extrait d'entretien avec un des plus proches conseiller du Premier ministre du Vanuatu va dans ce sens :
" Je pense que le grand problème des donneurs qui viennent à Vanuatu est qu'ils apportent leurs consul- tants, et ces consultants récoltent des informations qu'ils ramènent dans leurs pays pour exposer tel ou tel besoin du Vanuatu. Après ça, ils reviennent avec leurs idées et négocient avec le gouvernement. Ils disent "en fonction de nos recherches et de notre expertise, voilà ce dont vous avez besoin". Et c'est toujours comme ça que ça se passe, les suggestions des donneurs mènent à ce que le gouvernement formule un besoin en un budget d'aide au développement, basé sur ce que leurs consultants ont récolté comme informations. Ce qui conduit à ce que le gouvernement ne mène pas ses propres recherches (ou expertises) ou diagnostics de l'aide nécessaire, même si les idées des donneurs sont bonnes. Le gouvernement n'a pas réellement... il ne met pas trop d'emphase à l'appropriation de ce type

la sociologie des organisations, de l'analyse des politiques publiques et de la socio anthropologie du développement. Cependant, les usages pratiques qu’en font les professionnels du développement sont assez éloignés des prétentions théoriques du modèle. Son utilité dans la sélection ou dans l'évaluation des projets rencontre un large scepticisme, mais tous s'accordent à reconnaître qu'il peut permettre un contrôle de cohérence dans la conception des projets (Giovalucchi et al., 2009 : 1).

22. «J'appelle capital symbolique n'importe quelle espèce de capital (économique, culturel, scolaire ou social) lorsqu'elle est perçue selon des catégories de perception, des principes de vision et de division, des systèmes de classement, des schèmes classificatoires, des schèmes cognitifs, qui sont, au moins pour une part, le produit de l'incorporation des structures objectives du champ considéré, c’est-à-dire de la structure de la distribution du capital dans le champ considéré. » (Bourdieu : 1994, 161).

23. Létude des écarts entre la conception d'un projet et ses résultats finaux (ou implementation gap) a conduit à l'institution d'implementation studies qui offrent des analyses tant sur la mise en œuvre des politiques publiques au "Nord " que des projets au «Sud » (Pressman et al. 1973; Barrett, 2004 ; Nilsen et al., 2013). 
de projets... Bien souvent, de gros montants d'argent arrivent dans le pays et ils viennent avec leur propre personnel, tu le sais... Et on regarde ces gros projets mis en œuvre, dont beaucoup d'entre eux, sont pour le renforcement des capacités [...]. Mais le problème est que nos besoins sont vus d'une perspective extérieure, il n'y a donc pas vraiment d'appropriation. " (Extrait d'entretien du 05/08/2013)

Ce témoignage illustre que même au plus haut niveau du gouvernement, les donneurs tendent à orienter la mise en œuvre de projets d'aide au développement en fonction de leur expertise et donc, de leurs représentations. Lors d'un entretien mené avec un spécialiste de l'agriculture à Vanuatu, celui-ci a tenu un discours proche de celui de ce conseiller ministériel. Il émit des doutes sur l'assurance des experts étrangers d'être les détenteurs de "savoirs légitimes " et ainsi pouvoir orienter les politiques publiques. La discussion portait sur la politique de soutien à l'agriculture par les donneurs depuis l'indépendance du Vanuatu, notamment sur l'absence d'aide à la production de kava.

"Quand tu es invité aux bonnes tables, l'agronome qui vient de Paris, il fait de l'ethnocentrisme. Ils disent "ces gens-là [les Mélanésiens] doivent faire de l'agriculture digne. De l'agriculture digne, c'est des produits qu'on aime. Il est hors de question qu'ils boivent du kava. Vous n'y pensez pas, ça a un mauvais goût, c'est une drogue abominable, comment vous pouvez encourager le kava !?”. Ils [les donneurs] n'ont jamais misé un euro sur le kava alors que la culture de cette plante est le succès de l'agriculture de ces quarante dernières années. Ça permet de renvoyer l'argent des fonctionnaires des villes vers les îles, c'est cultivé selon des systèmes familiaux, avec des jardins traditionnels, c'est extrêmement souple au niveau du calendrier, donc c'est extrêmement mélanésien, et ça plait. L'absence d'aide pour le kava, c'est le miroir de l'échec de l'aide occidentale à l'agriculture. Ça n'a jamais été aidé alors que c'est la plante qui a réussi malgré toutes les visions qu'avaient les agronomes qui arrivaient de Paris sur ce qu'il fallait faire en matière d'agriculture ici. Ils [les Mélanésiens] n’ont pas non plus été très bien aidés [...]» (Extrait d'entretien du 16/05/2012)

Depuis l'indépendance du Vanuatu, la culture du kava, plante qui symbolise le patrimoine traditionnel de ses habitants, n'a donc pas été aidée ou subventionnée par les bailleurs de fonds. Selon notre interlocuteur, cette situation s'explique par le fait que la plupart des agronomes impliqués dans la mise en œuvre des projets d'aide au développement ont leur propre vision de ce que doit être l'agriculture moderne, sans se préoccuper réellement du contexte local. Par ailleurs, la méthodologie de gestion de projet participe peu à ce que ces experts prennent en considération ces réalités locales. En effet, limitée dans le temps, la durée des missions des consultants varie généralement de quelques jours à maximum quelques semaines. Ce facteur leur offre donc peu de temps pour appréhender les spécificités du contexte d'intervention ${ }^{24}$.

Ce recours massif à des consultants étrangers s'explique par les ressources humaines limitées en experts locaux spécialisés sur les secteurs traditionnels de l'aide (santé, éducation, sécurité alimentaire, infrastructures, etc.) et en courtage de projets internationaux. Cette situation est notamment due à l'absence de formations supérieures en études sur le développement ou en gestion de projet dans l'archipel ${ }^{25}$. Le recours à des spécialistes de nationalité étrangère pour définir les priorités locales et les façons d'y répondre font donc office de règle. Cette problématique prend un relief particulier en ce qui concerne la lutte contre le changement climatique, un domaine emblématique du décalage entre les priorités des donneurs et celles des bénéficiaires de l'aide ainsi que de ses effets secondaires et structurants.

\section{La priorité de la lutte contre le changement climatique}

Aujourd'hui, à Vanuatu et dans le Pacifique, une des principales priorités, le secteur privilégié et qui bénéficie d'importants fonds d'aide au développement, est la lutte et l'adaptation face au changement climatique. Le géographe JeanChristophe Gay fait partie des voix critiques du phénomène de concentration de l'aide au développement pour cette priorité toujours plus exclusive. Ainsi, dans un article récent, l'auteur analyse

« le débat actuel sur les conséquences du réchauffement climatique et démontr[e] l'instrumentalisation

24. Claude Arditi offre un témoignage symptomatique de "descentes sur le terrain " considérées comme de simples formalités par des « experts galops» (2005).

25. nt l'accès à l'éducation, la scolarisation atteint des degrés élevés pour la région, de l'ordre de $90 \%$ pour la classe d'âge des enfants de 12 ans. Ce taux décline de manière drastique pour les écoliers plus âgés $(50 \%$ à 16 ans et $30 \%$ à 18 ans) ; l'accès aux études supérieures demeure le plus souvent l'apanage d'une élite (5\%) (vnso, 2010 : 23). Toutefois, la contrepartie à cette attention portée à la formation est une quête généralisée pour trouver l'argent nécessaire au règlement des frais de scolarité. Leur coût économique moyen, premier poste de dépense pour la plupart des familles, a largement contribué au renforcement de la monétarisation de l'économie de subsistance dans les zones rurales et, par extension, à leur paupérisation en termes d'économie formelle. Les capacités de scolarisation des enfants impliquent fréquemment une émigration vers Vila ou vers l'étranger dans le cadre d'emplois saisonniers ou d'embauches sur les flottilles de pêche étrangères." (Tabani, $2011: 232)$. 
des petits États insulaires par une coalition hétéroclite d'experts, d'activistes, de journalistes, de célébrités ou d'hommes politiques. »(Gay, 2014: 1)

\section{L'auteur ajoute également que :}

"le spectre de la montée du niveau de la mer et de la possible disparition de ces îles, avec son lot de "réfugiés climatiques", devient une véritable rente pour des États insulaires se posant comme victime du développement dans le dessein d'obtenir des compensations. » (Gay, $2014: 1$ )

À Vanuatu, pour une meilleure coordination des budgets subventionnés par les donneurs, en 2012, furent créés un ministère du Changement climatique et de Réduction des risques naturels et un comité national qui coordonne les activités menées dans ce secteur: le National Advisory Board on Climate Change and Disaster Risk Reduction $(\mathrm{NAB})^{26}$. Si bien que, le 31 mai 2013, un lecteur du journal Vanuatu Daily Post demanda à être éclairé sur l'impact des nombreuses initiatives supervisées par le NAB. Ce courrier se rapproche de nombreux autres témoignages de professionnels du développement avec lesquels nous avons pu nous entretenir au sujet du déferlement de fonds pour ce secteur dans l'archipel accompagnés de leurs myriades d'experts étrangers. Voici le contenu de cette lettre :

\section{"Cher Éditeur,}

Je vous écris pour vous demander que font les personnes travaillant au bureau du changement climatique pour s'occuper de cette question? Les scientifiques rendent confuses toutes ces questions concernant ce phénomène. À Vanuatu, nous en entendons parler depuis les années 1990. [...] J'entends parler du changement climatique comme quelque chose venant nous submerger alors que finalement rien ne se passe. Quand vous en parlez aux personnes des îles, ils ne savent pas non plus de quoi vous parlez. J'ai entendu dire que le bureau du changement climatique se réunissait très souvent pour discuter de ces nombreux aspects. Mais, savent-ils trouver une solution pour répondre à ses enjeux ou s'amusent-ils juste avec l'argent que leur donnent les donneurs? Apportez de réels projets sur le terrain pour prouver aux Ni-Vanuatu que vous êtes des scientifiques du changement climatique. Assez de parler pour ne rien dire et d'enchainer réunion après réunion pour quelque chose qui n'existe pas (ou que l'on ne voit pas). Je souhaite parler de ce sujet du changement climatique pour que l'on puisse ouvrir le débat.

\section{Stuart,}

Parc de l'indépendance

Port-Vila" ('Tumas toktok abaot climate change', courrier des lecteurs, Dailypost, 31/05/2013, trad. A. Hochet)

Une réponse à ces questions fut publiée la semaine suivante dans le courrier du lecteur, accompagnée d'une page entière de descriptions des projets mis en place et coordonnés par le $\mathrm{NAB}^{27}$. La pluralité des partenaires institutionnels locaux était présentée, ainsi que les nombreux domaines d'action au sein desquels les diverses parties prenantes interviennent. La répétition de onze acronymes ou sigles différents, utilisés soixante-huit fois en une seule page ${ }^{28}$, reflétait la diversité des organisations engagées dans ce champ, en plus d'en complexifier la lecture. Cet article faisait cependant l'impasse sur l'origine des fonds et surtout sur les montants considérables déboursés à l'échelle du pays pour la lutte contre le changement climatique ${ }^{29}$. Avec une emphase mise sur l'implication des organisations locales, les activités menées étaient ainsi énumérées :

«73 différents produits ont été créés pour informer la population sur les effets du changement climatique et des risques de désastres dont un éventail de brochures, de posters, de spots publicitaires (radio, télévision, journaux), d'affiches, de présentations scolaires, de sensibilisations à des événements, de DVD et autres supports internet permettant de disséminer l'information sur les effets du changement climatique [...] L'achat de voitures, de motos, d'un bateau et de matériel médical pour le département de l'Agriculture $[. .$.$] La reconstruction de routes, la restauration$ de bâtiments, la rénovation de laboratoires, d'équi-

26. Les objectifs du NAB sont "d'agir comme l'institution suprême de conseil et de la mise en œuvre de politiques publiques en matière de réduction des risques face à des désastres et du changement climatique " (Trad. A. Hochet). Il est coprésidé par les directeurs du Vanuatu Meteorological and Geo-hazard Departement (vMGD) et du Natural Disaster Management Office (NDMO) et les membres du comité directeur sont présentés en tant que "senior representatives » des agences gouvernementales et des ONG impliquées dans ces champs d'action (http://www.unescap.org/sites/default/files/ Vanuatu\%20Presentation\%2C\%20Rarotonga\%20workshop\%20sep2013.pdf, dernier accès le 17/02/2015).

27. 'Climate Change initiatives and activities in Vanuatu', Dailypost, 08/06/2013.

28. L'acronyme NAB : National Advisory Board on Climate Change and Disaster Risk Reduction fut répété 14 fois ; DRR : Disaster Risk Reduction 8; CC : Climate Change 7; CCA : Climate Change Adaptation 4; vMGD : Vanuatu Meteorological and Geo-Hazard Department 10; PMU : Program Management Unit 5; NDMO : National Disaster Management Office6; VHT : Vanuatu Humanitarian Team 4; varTC : Vanuatu Agricultural Research and Training Centre 1; PDCC : Provincial Disaster Climate Change Committee 4; CDCCC : Community Disaster Climate Change Committee 3; GIZ : 1.

29. Depuis sa création, le NAB a absorbé plusieurs millions d'euros. Selon une source interne, en juin 2013, tous projets confondus, le NAB coordonnait 4 milliards de vatu (32 millions d'euros) dans la lutte contre le changement climatique, soit près d'un quart du budget annuel du gouvernement vanuatais (120 millions d'euros). Difficilement accessibles, les responsables du NAB n'ont pas répondu à nos sollicitations d'entretiens. D'après les documents officiels à notre disposition et les discussions d'agents impliqués dans les projets intervenant dans ce secteur, nous avons pu comptabiliser au moins 22 millions d'euros d'appuis financiers provenant des bailleurs de fonds : 18 millions d'euros de l'unEP/UndP/FAO/Banque mondiale confondus, 4 millions de l'Union européenne. 
pement de bureau, de matériel de jardinage, de multiplication de plantes nutritives et résilientes face au changement climatique et de leur distribution aux horticulteurs, l'achat et l'installation de plus de 400 réservoirs d'eau de pluie, de formation à la plomberie [...] et de la mise en place d'un centre d'urgence et d'un plan d'évacuation en cas de catastrophe naturelle. " (Ibid., trad. A. Hochet)

La lutte contre le changement climatique à Vanuatu use de la notion d' "adaptation" (ou résilience) comme d'un slogan à l'égard des populations locales afin de les sensibiliser et leur permettre de contrer les effets les plus dévastateurs des désastres prédits par les scientifiques. Mais dans l'immédiat, elle permet surtout à de nombreuses institutions publiques locales de trouver des fonds supplémentaires à leur budget annuel. L'attention portée par les organisations locales aux questions de changement climatique est ainsi avant tout motivée par les sommes investies par les donneurs dans ce domaine. Il faut dire que ces derniers ne lésinent pas sur leurs frais de communication. Omniprésente et surmédiatisée, la lutte contre le changement climatique souffre alors d'une survisibilité menant à des confusions sur ses impacts. À titre d'exemple, dans son édition du 8 juin 2013, un article du Vanuatu Daily Post rapportait que l'état des routes à Port-Vila était mauvais, du fait, d'après un haut fonctionnaire de l'administration, du changement climatique : "le Directeur général a dit qu’à cause du changement climatique, il y a trop de pluie à Vanuatu aujourd'hui ». En d'autres mots, le mauvais état des routes de Port-Vila serait dû à une surabondance de pluies, elles-mêmes dues au changement climatique et non à l'absence de leur maintenance.

Ailleurs, une coopérante salariée d'une ambassade présente à Port-Vila évoque l'emballement médiatique autour de la question du changement climatique qui participe à la même dynamique générale de concentration des efforts des donneurs et des agents locaux du développement sur cette thématique au détriment d'autres secteurs prioritaires :

"Je ne sais pas si tu connais le cas de Tegua, au Torres. C'est une petite île, qui a soi-disant été classée comme île où il y a eu les premiers réfugiés climatiques, première île victime de la montée des eaux en 2005. La BBC s'est quand même rendue là-bas pour faire un reportage sur ces pauvres gens, ils doivent être 80 à habiter sur l'île. Et donc voilà, la BBC a été là-bas, toutes les caméras du monde étaient braquées sur ces pauvres gens. Donc, quand tu vas là-bas, il y a des aides de partout... aujourd'hui, j'ai encore des journalistes qui m'appellent en me disant «j'aimerais aller à Tegua pour aller filmer, pour aller faire un reportage sur les gens de là-bas». Mais, en fait, ils ont bénéficié de tellement d'aide - j'y suis allée il y a pas longtemps -, ils ont reçu au moins huit réservoirs d'eau de 6000 litres. Ils en ont tellement - parce qu'on leur a donné des réservoirs d'eau, mais on ne leur a pas donné des toiles pour récolter l'eau, donc du coup, ils doivent en avoir un ou deux maximum qui servent vraiment - , ils sont là pour que les touristes les voient... Bref, ils en ont en trop et ils ne savent pas quoi en faire. Je crois que c'est Canada Aid qui a financé ça. Ils se sont dits "au moins, on repart, mais ils ont de l'eau". Mais, quand tu discutes avec les villageois, ils t'expliquent qu'ils n'ont pas du tout eu de montée des eaux, c'est juste qu'il y a eu un mini tsunami, et comme le village est très près de la plage, dans une zone basse par rapport au niveau de l'eau, ce n'était pas une grosse vague, mais l'eau qui est montée et qui a tout inondé. C'est resté là deux ou trois semaines. Donc effectivement, ils ont été inondés, ils ont dit que c'était le changement climatique, mais rien à voir avec la montée des eaux... enfin, le changement climatique... il y a aussi un enfoncement naturel des îles... donc là, tu te dis... c'est ça qui me gêne, j'aimerais bien plus qu'on mette l'accent sur la formation [...]. » (Extrait d'entretien du 05/08/2014).

À ce stade, il apparaît que le facteur culturel s'avère peu pertinent pour rendre compte des dynamiques de définition et de mise en œuvre des politiques publiques dans le pays. À travers les témoignages restitués, le constat que les priorités nationales et sectorielles soient souvent orientées par l'entremise d'agents ou de structures extérieures se présente comme un critère déterminant pour comprendre les particularités (voire les dysfonctionnements) de l'action publique de l'Etat vanuatais. Cette tendance conduit selon nous à trois principaux types de réactions des structures locales. La première est l'absence d'appropriation des projets impulsés par les donneurs telle qu'évoquée par le conseiller du Premier ministre ou encore l'agronome. La deuxième est l'émergence de secteurs concentrant d'importants moyens financiers et donc l'attention des structures locales formant des "îlots d'efficacité " (Blundo, 2011b) telle que l'action du NAB. Et enfin, plus rarement, une résistance des structures locales vis-à-vis de projets perçus comme trop intrusifs dans les affaires locales. Ce dernier modèle semble d'autant plus valable lorsqu'on l'applique à l'ethnographie de l'institution nationale vanuataise qui a pour mission de préserver, protéger et promouvoir les différents aspects de la culture et s'avère donc un rouage essentiel de la politique culturelle nationale: le Centre Culturel du Vanuatu.

\section{Le Centre culturel du Vanuatu : un fragile équilibre entre volonté d'autonomie et priorités allogènes}

Notre expérience de collaborateur au sein de cette institution nous a permis de constater 


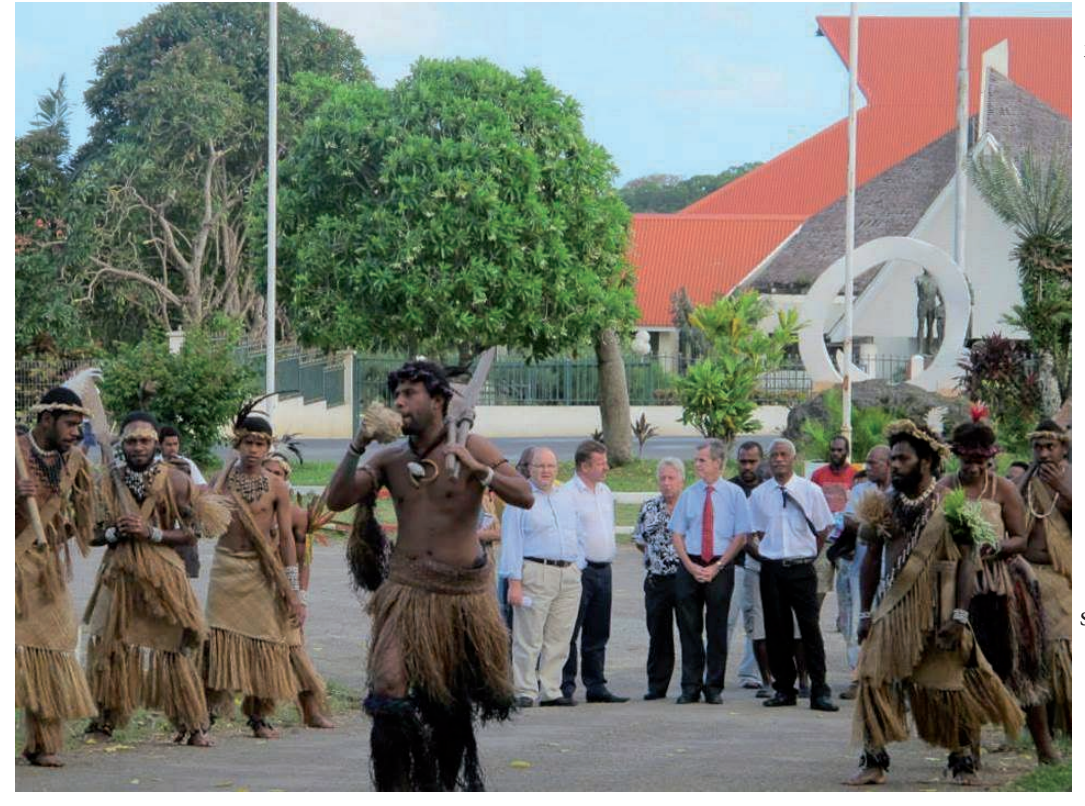

FIGURE 1. - Accueil de représentants officiels de bailleurs de fonds pour une cérémonie au Centre culturel du Vanuatu. Entrée du Centre Culturel du Vanuatu, Port-Vila, 2013 (C) Antoine Hochet)
Les bailleurs de fonds représentés sur l'illustration ont soutenu le Centre culturel du Vanuatu pour la publication de plusieurs ouvrages en sciences humaines et sociales sur le Vanuatu et le Pacifique. Au premier plan, figurent des danseurs traditionnels de l'île de Futuna. Au second plan, de gauche à droite, étaient présents le haut-commissaire australien, le chargé d'Affaires de la délégation de l'Union européenne à Vanuatu, le conseiller de coopération et d'action culturelle de

l'ambassade de France, l'ambassadeur de France, un salarié du Fonds Christensen, le président du Conseil national de la culture du Vanuatu. Au troisième plan, une statue en forme de dent de cochon fut érigée grâce au fonds de l'UE Afrique-Caraïbes-Pacifique. Au dernier plan, le Parlement du Vanuatu financé et construit avec le soutien de la Chine. que lorsque le Centre culturel du Vanuatu (ou Vanuatu Kaljoral Senta - vKs) disposait d'un budget afin de recueillir et de valoriser son patrimoine culturel, historique et artistique, les connaissances utiles pour l'adaptation au changement climatique ne figuraient pas comme prioritaires. Les ressources étaient effectivement avant tout dirigées d'une part, sur la formation de personnels capables de gérer des projets de développement, d'autre part, sur des questions sociales et politiques plus contextuelles, notamment celles de la tenure traditionnelle des terres ou, en d'autres termes, sur les questions de propriété et de préservation des moyens de production afin que les populations puissent continuer à assurer leur auto-subsistance.

Le projet Mama Graon, soutenu par l'Australie, était dédié à cette dernière thématique. Il avait pour objectif de renforcer les structures locales de gestion du secteur du foncier afin de résoudre les nombreux conflits locaux attenants à sa redistribution et son exploitation. Une des principales activités consistait à " recueillir " les savoirs traditionnels des populations rurales sur les différents systèmes de parenté, la pluralité des pratiques d'usage des terres ou ce qui est plus couramment nommé les savoirs coutumiers sur la gestion des terres. Mais, dès le lancement du projet, les coordinateurs de Mama Graon durent faire face à plusieurs obstacles. Le principal d'entre eux fut le Centre Culturel du Vanuatu.

Le Centre Culturel du Vanuatu et son réseau de fieldworkers ${ }^{30}$ avaient un rôle central à jouer dans la mise en œuvre de ce projet. Mais, la coordination et les postes de direction ont pour la plupart été attribués à des personnels expatriés ou à des chercheurs étrangers. Le Centre Culturel du Vanuatu devait alors mettre en œeuvre des activités définies en amont et supervisées par des décideurs étrangers, indépendamment du Conseil National de la Culture du Vanuatu (CNCV) qui fait pourtant autorité en matière de définition des politiques culturelles et de l'étude des coutumes locales ${ }^{31}$. Ainsi, le vKs et le CNCV ne disposaient que d'une faible marge de manœuvre pour infléchir les orientations de certaines

30. «Le réseau des filwoka se réunit annuellement au Centre culturel de Port-Vila pour discuter des différents aspects de la culture du Vanuatu. Lors de ces réunions, un sujet spécifique est traité (par exemple : l'alimentation, l'architecture, la gouvernance coutumière, les chants, etc.) et un nouveau est choisi, laissant alors aux filwoka une année pour accumuler des données pour la réunion suivante. À l'heure actuelle, environ 150 filwoka hommes et femmes sont membres de ce réseau basé sur le volontariat. Tous les ans, les présentations et débats ayant lieu lors de leurs rassemblements sont enregistrés puis ensuite conservés au sein de la section d'Archives audiovisuelle et Sonore du vKs, constituant ainsi une base de données unique et précieuse pour le patrimoine culturel national vanuatais. " (Hochet, 2012).

31. Le Conseil national de la Culture du Vanuatu est l'entité déterminant la politique du Centre culturel du Vanuatu. Il est «composé d'un représentant du ministère de l'Intérieur, du Conseil national des Chefs ou Malvatumauri (lui-même également sous l'égide du ministère de la Justice), du Conseil national des Femmes, du représentant du personnel du vks et enfin, de son directeur, nommé par le ministère de l'Intérieur. Le CNCV se réunit au minimum deux fois par an, cela laissant une importante part de liberté à son directeur dans la gestion des affaires courantes. » (Ibid. : 105) 
activités du projet. Des tensions concernant la coordination de ces activités émergèrent donc avant même leur mise en œuvre.

La mission du Centre Culturel et de ses fieldworkers consistait à récolter des informations sur les différents systèmes coutumiers de tenures foncières. Mais, la question de savoir à qui appartenaient les données collectées divisa les responsables du projet et le directeur du VKS, Marcelin Abong. Joël Simo, personnage de notoriété à Vanuatu, militant pour la défense des droits fonciers mélanésien et porte-parole $\mathrm{du}$ Land Desk ${ }^{32}$, nous a décrit en détail une des étapes de cette négociation dont il fut témoin :

«Ils [les responsables du projet Mama Graon] sont venus l'année dernière, pour poser leurs conditions. Mais Marcelin leur a dit directement: "non, c'est nous qui décidons pourquoi vous donnez de l'argent, vous ne déciderez pas pour nous". Mais ils sont puissants... Ils sont revenus quelques jours plus tard. Marcelin ne les a même pas invités à s'assoir, il a dit qu'il ne souhaitait pas coopérer, qu'il avait peur qu'ils veuillent nous contrôler, qu'il appréhendait. Toutes ces informations, ce n'est pas pour nous, c'est pour eux, ces gens-là... et s'ils nous contrôlent, ils pourront avoir accès à toutes ces informations-là. C'est ce problème-là que moi j'appréhende [...] Ils voulaient financer tous les filwoka wokshop." (extrait d'entretien du 13/09/201).

Ce témoignage de Joël Simo met ici en évidence la puissance des moyens financiers dont disposent les donneurs pour inciter les administrations locales à soutenir des activités, selon leurs conditions ${ }^{33}$. Déterminer qui disposait de l'accès et des droits d'exploitation des données collectées était décisif et fut la pomme de discorde entre les participants. Cette situation est notamment due au fait qu'historiquement, le Centre culturel centralise ce type d'informations confidentielles. Depuis ses origines le vKS dispose en effet d'une légitimité incontestable pour tout ce qui a trait à la centralisation d'informations concernant la gouvernance coutumière et à la gestion traditionnelle du foncier. Les informations archivées, dans ses locaux, et sur différents supports (audiovisuels, sonores, photographiques, livresques, etc.), peuvent être très sensibles dans la mesure où elles sont susceptibles d'être utilisées en tant que preuves lors de procès pour litiges fonciers. Or, en compilant et en exploitant de telles informations, Mama Graon se substituait au rôle imparti au vKs, garant de la confidentialité de ces données depuis l'indépendance du pays.

Par ailleurs, le Centre culturel du Vanuatu ne fut pas la seule institution à opposer une résistance au projet Mama Graon. Gratien Alguet, président du Conseil national des Chefs du Vanuatu (Malvatumauri) jusqu’à 2013, publia une lettre ouverte à l'attention du Premier ministre du Vanuatu pour dénoncer les travers du projet ainsi que la sélection d'un cabinet d'expertise étranger pour le coordonner : Land Equity International ltd (LEI).

«Premièrement [le projet] a été conçu par une entreprise étrangère en suivant une requête dont le Malvatumauri n'était pas informé ; deuxièmement, le Malvatumauri ne faisait pas partie de l'équipe qui a conçu le projet. D'après ce que je comprends, Ausaid a financé un cabinet qui a pris seulement six mois pour penser ce projet. [...] Quand la conception fut finalisée, le donneur a signé un accord de financement avec cette même entreprise australienne pour mettre en œuvre le projet. Par conséquent, cette entreprise s'est approprié le projet Mama Graon. [...] La question est de savoir 'pourquoi les donneurs veulent que Land Equity International fasse le travail du Malvatumauri? L'énorme budget qui a été dépensé pour LEI aurait directement pu aller à développer les capacités administratives du Malvatumauri et des conseils des Chefs des Îles à travers tout le Vanuatu et leur permettre de gérer Mama Graon. Puisque Lei est une entreprise australienne, ils ont automatiquement la mission de respecter et de soutenir la plupart des politiques et des valeurs de l'Australie en mettant en ouvre Mama Graon. [...] Je recommande fortement au gouvernement d'ordonner à LeI de suspendre ses missions afin d'effectuer une revue urgente de la conception et des arrangements de l'implémentation du programme. » (Dailypost, 07/07/2012, trad. A. Hochet)

Le président Alguet critiquait ouvertement la politique extérieure lancée en 2008 par l'Australie sur la question foncière dans le Pacifique - plus connue sous le nom de Pacific Land Program. Ce programme régional visait à soutenir des réformes dans les États insulaires océaniens afin de renforcer les systèmes de tenure foncière. L'impression d'un détournement des objectifs initiaux du processus de réforme

32. Le Land Desk est une "structure comprenant quatre salariés à temps complet, intégralement financée par la Fondation Christensen (FC), [qui] est chargée d'effectuer une veille informationnelle et des campagnes de sensibilisation (ateliers, séminaires, etc.) principalement à destination des populations rurales en vue de les informer sur leurs droits fonciers et sur l'aliénation des terres en cours depuis ces dernières décennies dans l'archipel " (Hochet, 2012 : 112). Joël Simo est le responsable du Land Desk et également membre fondateur et actif de la Melanesian Indigenous Land Defence Alliance (MILDA) qui regroupe des activistes mélanésiens œuvrant pour la protection et à la promotion des droits fonciers autochtones. Joël fut un collègue de travail pendant toute la durée de notre collaboration avec le Centre culturel du Vanuatu.

33. Le budget du programme Mama Graon était de 20,3 millions de dollars australiens (http://aid.dfat.gov.au/countries/ pacific/vanuatu/documents/vanuatu-land-program-joint-review-plan.pdf, dernier accès le 17/02/2015). Â titre comparatif, le budget annuel du gouvernement du Vanuatu en 2013 est d'environ 120 millions de dollars australiens, celui du Centre culturel du Vanuatu, de 250 millions de dollars australiens. 
nationale du foncier, initialement impulsé par le gouvernement vanuatais, fut renforcée par la sélection du cabinet de consultance étranger Land Equity International. Le recrutement de ces experts créa une vague de mécontentement chez les spécialistes locaux de la question foncière, Joel Simo en témoigne :

"J. : Quel cabinet ont-ils choisi? Qui est ce groupe de personnes qui dirige le projet Mama Graon? Ils n'ont pas autorisé un cabinet du Vanuatu, pas un cabinet national. Je pense que c'est vraiment une grossière erreur parce que ce sont les gens du Vanuatu qui sont les experts de leurs terres. Ce ne sont pas les étrangers. Non. Donc, ils ont fait l'appel d'offres à l'international, Land Equity, c'est un cabinet australien.

H. : Mais, ils avaient déjà travaillé ici auparavant ? J. : Ils avaient travaillé ici. Ils sont venus faire une étude qui a débouché sur un rapport. J'avais même collaboré avec eux pour qu'on fasse une revue des lois sur le foncier $[\ldots]$. Quand ils ont remporté l'appel projet, ils sont devenus alors ceux qui s'occupèrent de la gestion coutumière des terres à Vanuatu. S'ils viennent pour produire des analyses et des rapports sur les terres qui sont déjà enregistrées, ça, ça fait sens, parce que c'est le régime du système étranger, à l'occidentale, c'est calqué sur le système juridique étranger. Mais ils viennent également pour dire comment Vanuatu doit gérer ses terres coutumières. C'est là que ça m'interroge beaucoup, parce que les experts des terres coutumières à Vanuatu, ce sont les Vanuatais. Les gens qui vivent sur ces terres, ce ne sont pas Land Equity ni Mama Graon. Donc, quand ils ont intégré le projet, au lieu de s'occuper des résolutions du Land Summit de 2006, ils sont allés là-haut [au Malvatumauri], ils ont fait des déclarations, ils ont demandé au Vks qu'il collabore avec eux. Ils sont venus me voir au Land Desk pour me dire que nous devions faire des recherches sur la gestion des terres coutumières à Vanuatu. J'y ai beaucoup réfléchi et j'appréhende à l'idée de collaborer avec eux. Parce que c'est faire une recherche sur la vie des Vanuatais, et la connaissance, c'est le pouvoir [...]. Si je connais ton passé, je sais qui tu es, je sais ce que tu es... [...] le problème, c'est que le savoir est pour les gens, ce n'est pas à moi de l'exposer. Ce qui fait que je n'ai pas pris le risque. Ils ont essayé de venir à l'intérieur de notre structure, mais moi, j'appréhende vraiment de ce qui pourrait être fait des informations qui, si elles sortaient au grand public, auraient des conséquences néfastes. Parce que toutes ces informations-là, elles sont pour les gens, c'est leur vie. Si on fait une erreur, les gens, n'importe quelle entreprise pourrait même les utiliser pour leur nuire, pour les tenir... Donc, moi, nous au VKS, on est assez sceptiques d'aller plus loin avec eux. » (Extrait d'entretien du 13/09/2012)

Comparé par un cadre du bureau du Malvatumauri à une "fleur toute mignonne arrivant avec son petit baluchon " ou encore à " un père noël arrivant avec plein de cadeaux " (extrait d'entretien du 30/06/2013), le programme Mama Graon avait beaucoup à offrir, mais l'image de l'Australie, beaucoup à perdre, tant la question de la tenure foncière est sensible à Vanuatu. Un ambassadeur européen confirma cette idée. Lors d'une discussion informelle, celui-ci expliqua que son pays ne s'impliquait pas dans les questions de la tenure foncière dans l'archipel celles-ci étant jugées trop délicates par son administration en ce qu'elles risqueraient de compromettre les relations diplomatiques entre les deux nations (extrait d'entretien du 01/09/2012). Lors d'un entretien avec un consultant mandaté afin de fournir une assistance technique à la mise en ouvre de Mama Graon, celui-ci était également conscient des enjeux qu'induit toute implication de donneurs dans les politiques de gestion des terres :

"Disons que le gros capital ici, le seul intérêt - mais il n'y a pas de gros capitaux ici - le seul intérêt, c'est la terre. Aujourd'hui, l'argent qui s'investit au Vanuatu, il s'investit dans la terre. On achète un terrain, on subdivise, on construit, on vend, on loue. On trouve un autre terrain, on refait, etc., etc. On fait rentrer de la valeur comme ça. On crée de la valeur, avec la force de l'investissement foncier. C'est ça qui se passe, c'est la seule ressource, pour les investisseurs extérieurs. Bon, il y en a bien qui essaient de faire des poulaillers, des petites industries, bien sûr, évidemment, le coprah, le cacao... Mais, l'essentiel des investissements, pour moi ici, c'est la terre. Ce qui nous différencie beaucoup de la Papouasie par exemple, où là, ce sont les mines. » (Extrait d'entretien du 12/11/2012)

L'expert-consultant évoqua les trois principaux objectifs du projet Mama Graon et les difficultés pour y parvenir. Le premier était d'offrir un appui aux opérations foncières modernes, ou en d'autres termes, d'améliorer le système administratif d'enregistrement déjà existant. Le deuxième aspect, qu'il considérait comme plus "complexe et intéressant", concerne les procédures de contractualisation de baux,

" c'est-à-dire, le moment où un terrain donné passe du domaine coutumier, de propriété traditionnelle coutumière sans papier, collective, avec des droits complexes et qui ne sont pas définis par des lois écrites, comment on passe d'un bout de terrain donné qui est sous ce régime à un régime moderne, c'est-àdire, "tracé, dessiné, cadastré» et avec un propriétaire nommé, sûr. Privé ou public d'ailleurs. Comment faire passer un terrain du statut ancien au statut nouveau. Ça, c'est la procédure de 'lease'. Parce qu'une fois qu'il est rentré dans le système, là, ça y est, ça devient un bien marchand, et là, ceux qui s'imaginent que c'est différent se trompent. [...] Enfin, le troisième, il est jamais dit pour ça... enfin, si, c'est dit, mais c'est plus compliqué, c'est indépendamment d'une procédure qui va au lease. Parce qu'aujourd'hui c'est le seul moyen, en fait, la seule façon pour un individu ou un groupe de sceller son droit foncier, c'est de passer par un bail. En fait, si tu loues ton terrain à quelqu'un d'autre, on reconnait ta propriété. Tant que tu ne l'as pas fait, on ne reconnaît pas ta propriété. On le reconnaît dans la coutume, mais c'est incertain, ce n'est pas 
écrit. Il n'y a pas un banquier qui va croire ça. Donc, le troisième aspect c'est comment faire en sorte que les droits fonciers coutumiers soient éclaircis et protégés. Et là, on est dans le flou intégral, parce qu'on peut tout faire... » (Extrait d'entretien du 03/08/2013)

Si l'on suit le raisonnement du président Alguet à la lumière du témoignage de cet expert, le " flou intégral » dans la procédure à suivre afin que "les droits fonciers coutumiers soient éclaircis et protégés " s'expliquerait en grande partie par la précipitation des experts du développement à concevoir le projet. Son principal argument reposait effectivement sur la courte durée, six mois, consacrée à sa conception afin de définir un programme quinquennal aux fortes implications politiques, économiques et sociales. Mais selon nous, l'impossibilité de parvenir à un accord pour déterminer qui détenait les droits d'exploitation des informations collectées sur les modes locaux de gouvernance foncière explique en premier lieu l'opposition des structures locales à la mise en œuvre du projet (notamment par le vKs), ce qui conduisit de nombreuses personnalités à prendre position publiquement. Finalement, Mama Graon fut abandonné à miparcours (juin 2013).

\section{Conclusion}

Par les témoignages recueillis et présentés dans cet article, notre intention fut de montrer que l'analyse des effets structurants de l'aide au développement se présente comme une approche plus féconde que l'explication culturaliste pour rendre compte du fonctionnement de l'État vanuatais et de ses administrations locales. Nos observations, discours et matériaux récoltés à Vanuatu convergent davantage vers l'idée que les priorités des donneurs ont une influence déterminante sur la mise en œuvre d'action publique en coopération avec les structures récipiendaires de l'aide. Ce phénomène entraîne chez les administrations locales des réactions variables : absence d'appropriation, émergence d'îlots d'efficacité dans certains secteurs ou, plus rarement, des résistances. Cette résistance reste selon nous peu représentative de la dynamique globale de l'aide observée sur le territoire vanuatais. L'abandon du projet Mama Graon fait en effet figure d'exception et ne fut possible que grâce à l'appui d'un réseau de ressources humaines diversifiées, proches du Centre culturel et sensibilisées aux enjeux complexes de la tenure foncière (réseau de fieldworkers, hauts fonctionnaires locaux, activistes de la société civile, etc.).

A Vanuatu, ces effets paradoxaux de l'aide peuvent s'expliquer par le faible nombre de ressources humaines locales, expertes et institutionnellement légitimes auprès des bailleurs de fonds pour les orienter afin qu'ils adaptent davantage leurs interventions au contexte local. L'accès aux savoirs "légitimes" s'avère effectivement difficile pour les $\mathrm{Ni}$ Vanuatu - bien que les frais d'éducation soient le premier poste de dépenses des foyers vanuatais. À la nécessité de s'expatrier pour suivre des formations supérieures et accéder aux " régimes de savoirs » des experts du monde du développement, s'ajoutent les coûts induits. Selon nous, cette configuration freine alors l'émergence d' "élites " scientifiques ou d'experts locaux qui pourraient développer un point de vue critique sur les modalités de l'aide ou proposer des modèles alternatifs et contextualisés de développement.

Ici encore, une explication culturaliste du désintérêt des populations mélanésiennes à accéder à ce type de connaissance ne semble pas non plus pertinente pour expliquer le faible nombre d'experts locaux légitimes auprès des donneurs présents dans le pays. En effet, pour Lindstrom, l'accès à l'information se présente comme une des caractéristiques spécifiques de la structuration du pouvoir au sein des sociétés traditionnelles qu'il a pu observer à Vanuatu. À l'instar de Joel Simo qui soulignait les liens intrinsèques entre le savoir et le pouvoir, Lindstrom a également mis en lumière le "pouvoir de la connaissance », considérée comme ressource symbolique déterminante dans son analyse ethnographique de la société de Tanna, une des îles du Vanuatu. On y apprend que "les insulaires savent qu'ils vivent dans des sociétés de l'information" (Lindstrom, 1990 : XIV; notre trad) dès lors considérées comme un "marché de la connaissance" (Ibid. : 22). Ce «marché de la connaissance» mériterait sans aucun doute davantage d'attention, non seulement des donneurs qui tendent davantage à privilégier ce qu'ils considèrent comme des priorités parfois peu en lien avec les enjeux socio-politiques locaux, mais également des ethnographes pour réfuter des discours culturalistes toujours persistants.

\section{BIBLIOGRAPHIE}

Anders Gerhart, 2003. An Ethnography of "Loan Arrangements" Between the Bretton Woods Institutions and the Government of Malawi : Good Governance as Technology, papier présenté au workshop Order and Disjuncture : The Organisation of Aid and Development ", Soas, Londres, 26-28 Septembre. 
Arditi Claude, 2005. Niger : chronique d'une évaluation censurée, Tiers-Monde 46, 184, pp. 861-881.

Argounès Fabrice, 2012. L'Australie : la tentation de la puissance régionale, Pouvoirs 141, 2, pp. 103-116.

BARrett Susan M., 2004. Implementation Studies: Time for a Revival? Personal Reflections on 20 Years of Implementation Studies, Public Administration 82, 2, pp. 249-262.

BAYART Jean-François, 1989. L'état en Afrique : la politique du ventre. Fayard.

—, 1996. L'illusion Identitaire, Fayard.

—, 1999. L'Afrique dans le monde : une histoire d'extraversion, Critique Internationale 5, 1, pp. 97-120.

Bhargava Rajeev, 2013. Overcoming the Epistemic Injustice of Colonialism, Global Policy 4 , 4, pp. 413-417.

Bierschenk Thomas, 2009. Anthropologie et développement. Historiciser et localiser les approches, Bulletin de l'Apad 31 (http://apad. revues.org/4065).

Bierschenk Thomas, Giorgio Blundo, Yannick Jaffré et Mahaman Tidjani Alou, 2007. Une anthropologie entre rigueur et engagement. Essais autour de l'oeuvre de Jean-Pierre Olivier de Sardan, Karthala.

Birdsall Nancy, 2004. Seven Deadly Sins: Reflections on Donor Failings, Working Paper 50, Center for Global Development, Washington.

Blundo Giorgio, 2011a. "Comme un ballon de foot". La gestion quotidienne des ressources humaines dans les services forestiers en afrique de l'ouest. Auf Dem Boden Der Tatsachen, Köln, Rüdiger Köppe Verlag (" Mainzer Beiträge Zur Afrikaforschung »), pp. 377-394.

—, 2011b. Une administration à deux vitesses projets de développement et construction de l'état au sahel, Cahiers d'études africaines 202203, pp. 427-452.

Bonhomme Julien, 2007. "Anthropologues embarqués», revue en ligne 'la vie des idées' (http://www.laviedesidees.fr/Anthropologues-embarques.html).

Bourdieu Pierre, 1994. Raisons pratiques : sur la théorie de l'action, Paris, Seuil.

Cantens Thomas, 2009. Être chef dans les douanes camerounaises, entre idéal-type, $t i t u$ lar chief et big katika, Afrique contemporaine 230, 2, pp. 83-100.
Carbonnier Gilles, 2010. L'aide au développement une fois de plus sous le feu de la critique. Revue internationale de politique de développement 1, pp. 141-147.

Cernea Michael, 1985. Putting People First: Sociological Variables in Rural Development, New York, Banque mondiale et Oxford University Press.

Chambers Robert, 1984. Rural Development: Putting the Last First, London-New York, Longman.

-, 1994. The Origins and Practice of Participatory Rural Appraisal. World Development 7, 2, pp. $953-969$.

Chappell David, 2005. "Africanization" in the Pacific: Blaming Others for Disorder in the Periphery?, Society for Comparative Studies in Society and History 47, 2, pp. 286-317.

Charnoz Olivier, 2010. Le discours mondial sur la "participation" et son émergence dans la protection de la biodiversité, Paris, AfD.

Coolidge Jacqueline et Susan Rose-Ackerman, 2000. Kleptocracy and Reform in African Regimes: Theory and Examples, in K.R Hope et B.C. Chikulo (éds) Corruption and Development in Africa. Lessons from Country Case-Stud$i e$, Houndmills et Basingtoke, Saint-Martin Press et Macmillan Press, pp. 57-86.

Darbon Dominique, 1990. L'État prédateur, Politique Africaine 39, pp. 37-45.

Escobar Arturo, 1995. Encountering Development: The Making and Unmaking of the Third World, Princeton, Princeton University Press.

Farrington John et Adrienne Martin, 1988. Farmer Participation in Agricultural Research: A Review of Concepts and Practices, ODI.

Fatton Robert, 1992. Predatory Rule: State and Civil Society in Africa, Colorado, Lynne Rienner Boulder.

Ferguson James, 1990. The Anti-Politics Machine: "Development," Depoliticization, and Bureancratic Power in Lesotho, Cambridge, Cambridge University Press.

FirTh Stewart, 2001. A Reflection on South Pacific Regional Security, Mid-2000 to Mid2001, The Journal of Pacific History 36, 3, pp. 277-283.

Fraenkel Jon, 2004. The Coming Anarchy in Oceania? A Critique of the "Africanisation" of the South Pacific Thesis, Commonwealth \& Comparative Politics 42, 1, pp. 1-34. 
GAY Jean-Christophe, 2014. Le réchauffement climatique: l'instrumentalisation des îles, L'Espace géographique 43, 1, pp. 81-89.

Geertz Clifford, 1973. The Interpretation of Cultures: Selected Essays, New-York, Basic Books.

Giovalucchi François et Jean-Pierre Olivier De Sardan, 2009. Planification, gestion et politique dans l'aide au développement: le cadre logique, outil et miroir des développeurs, Tiers-Monde 198, 2, pp. 383-406.

Goody Jack, 1992. Culture and Its Boundaries: A European View, Social Anthropology 1, pp. 9-32.

Grignon Claude et Jean-Claude Passeron, 1989. Le savant et le populaire : misérabilisme et populisme en sociologie et en littérature, Gallimard, Le Seuil.

Hobart Mark, 1993. An Anthropological Critique of Development: The Growth of Ignorance, London, New York, Routledge.

Hochet Antoine, 2012. Coopération et développement : Une étude du Centre culturel du Vanuatu, in A. Hochet et M. TABANi (éds), Cultures, sociétés et environnements à Vanuatu et dans le Pacifique, Port-Vila, Vks Publications, pp. 99-125.

Hugon Philippe, 2007. Retour sur une cinquantaine d'années d'économie du développement dans le tiers-monde, Tiers-Monde 191 (3), pp. 717-741.

JaFfré Yannick et Jean-Pierre Olivier de SARDAN, 2003. Une médecine inhospitalière: les difficiles relations entre soignants et soignés dans cinq capitales d'Afrique de l'Ouest, Paris, Marseille, Karthala-APAD.

Joseph Richard A., 1987. Democracy and Prebendal Politics in Nigeria: The Rise and Fall of the Second Republic, Cambridge-New York, Cambridge University Press.

Kuper Adam, 1999. Culture: The Anthropologists' Account, Cambridge, Mass, Harvard University Press.

Latouche Serge, 1989. L'occidentalisation du monde: essai sur la signification, la portée et les limites de l'uniformisation planétaire, Paris, La Découverte.

Latour Bruno, 1983. Comment redistribuer le Grand Partage ?, Revue de Synthèse 110, pp. 203-236.

LebLic Isabelle, 1993. Les Kanak face au développement : la voie étroite, Grenoble, Presses universitaires de Grenoble.
Lenclud Gérard, 1992. Le grand partage ou la tentation ethnologique, in G. Althabe, D. FABRE et G. LenClud (édis), Vers une ethnologie du présent, Paris, Msh, pp. 9-38.

Lewis David et David Mosse, 2006. Encountering Order and Disjuncture: Contemporary Anthropological Perspectives on the Organization of Development, Oxford Development Studies 34, 1, pp. 1-13.

Lindstrom Lamont, 1990. Knowledge and Power in a South Pacific Society, Washington DC, Smithsonian Institution Press.

Malinowski Bronislaw, 1929. Practical Anthropology, Africa 21, pp. 22-38.

Mauss Marcel, 1925. Essai sur le don: forme et raison de l'échange dans les sociétés archaïques, Librairie Félix Alcan.

Moss Todd, Gunilla Pettersson et Nicolas van de Walle, 2006. An Aid-Institutions Paradox. A Review Essay on Aid Dependency and State Building in Sub-Saharan Africa, Working Paper, 74, Washington DC, Center for Global Development.

Nilsen Per, Christian Staahl, Kerstin Roback et Paul Cairney, 2013. Never the Twain Shall Meet? A Comparison of Implementation Science and Policy Implementation Research, Implementation Science 63, 8, pp. 1-12.

Nugent Paul, 2010. States and Social Contracts in Africa, New Left Review 63, pp. 35-68.

Olivier de Sardan Jean-Pierre, 1995. Anthropologie et développement. Essai en socio-anthropologie du changement social, Paris-Marseille, Karthala.

—, 2010. Le Culturalisme Traditionaliste Africaniste, Cahiers d'Etudes africaines 198-199200, pp. 419-453.

PArk Taey-Gyun, 2006. W. W. Rostow et son discours sur l'économie en Corée du Sud dans les années 1960, Histoire, économie \& société $25^{\mathrm{e}}$ année 2, pp. 281-289.

Passeron Jean-Claude, 1990. Le raisonnement sociologique l'espace non-poppérien du raisonnement naturel, Paris, Nathan.

Pressman Jeffrey L. et Aaron B. Wildavsky, 1973. Implementation: How Great Expectations in Washington Are Dashed in Oakland: Or, Why It's Amazing That Federal Programs Work at All, This Being a Saga of the Economic Development Administration as Told by Two Sympathetic Observers Who Seek to Build Morals on a Foundation of Ruined Hopes, Berkeley, University of California Press. 
ReIlly Benjamin, 2000. The Africanisation of the South Pacific, Australian Journal of International Affairs 54, 3, pp. 261-268.

Reilly Benjamin et Robert Phillpot, 2002. "Making Democracy Work" in Papua New Guinea: Social Capital and Provincial Development in an Ethnically Fragmented Society, Asian Survey 42, 6, pp. 906-927.

Rist Gilbert, 1996. Le développement: histoire d'une croyance occidentale, Paris, Presses de la Fondation nationale des sciences politiques.

Rosтow Walt Whitman, 1963. Les étapes de la croissance économique, Paris, Éditions du Seuil.

SACHs Jeffrey, 2005. The End of Poverty: Economic Possibilities for Our Time, New York, Penguin Press.

SAHLINs Marshall, 1976. Culture and Practical Reason, Chicago, University of Chicago Press.

Schatzberg Michael G., 2001. Political Legitimacy in Middle Africa Father, Family, Food, Bloomington, Indiana University Press.

Sсотт James C., 1998. Seeing like a state: how certain schemes to improve the human condition have failed, New Haven, États-Unis Royaume-Uni.
Severino Jean-Michel et Olivier Ray, 2011. La fin de l'aide publique au développement : Mort et renaissance d'une politique publique globale, Revue d'économie du développement 25, 1, pp. 5-44.

Spivak Gayatri C., 1999. A Critique of Postcolonial Reason: Toward a History of the Vanishing Present, Cambridge, Harvard University Press.

Tabani Marc, 2011. Présentation. Vers un cinquantenaire de la République du Vanuatu, Journal de la Société des Océanistes 133, 2, pp. 229-240 (http://jso.revues.org/6459).

Tylor Edward B., 1958 [1871]. Primitive Culture, New York, Harper.

UnEsCo, 2005. Vers les sociétés du savoir: rapport mondial de l'UNESCO, Paris, Éditions de l'unEsCO.

VAnuatu National Statistical Office, 2011. 2009 Vanuatu National Population and Housing Census, vnso-spc.

Viltard Yves, 2006. L'étrange carrière du concept foucaldien d'épistémè en science politique, Raisons politiques 23, 3, pp. 193-202. 